\title{
Importance of serial changes in biomarkers in idiopathic pulmonary fibrosis
}

\author{
Akihiko Sokai ${ }^{1}$, Kiminobu Tanizawa ${ }^{2}$, Tomohiro Handa ${ }^{1}, K^{\prime}$ umiko Kanatani ${ }^{3}$, \\ Takeshi Kubo ${ }^{4}$, Kohei Ikezoe', Yoshinari Nakatsuka', Shinsaku Tokuda', \\ Toru Oga ${ }^{2}$, Toyohiro Hirai ${ }^{1}$, Sonoko Nagai ${ }^{5}$, Kazuo Chin $^{2}$ and Michiaki Mishima ${ }^{1}$
}

Affiliations: 'Dept of Respiratory Medicine, Graduate School of Medicine, Kyoto University, Kyoto, Japan. ${ }^{2}$ Dept of Respiratory Care and Sleep Control Medicine, Graduate School of Medicine, Kyoto University, Kyoto, Japan. ${ }^{3}$ Dept of Health Informatics, School of Public Health, Kyoto University, Kyoto, Japan. ${ }^{4}$ Dept of Diagnostic Imaging and Nuclear Medicine, Graduate School of Medicine, Kyoto University, Kyoto, Japan. ${ }^{5}$ Kyoto Central Clinic, Clinical Research Center, Kyoto, Japan.

Correspondence: Kiminobu Tanizawa, Dept of Respiratory Care and Sleep Control Medicine, Graduate School of Medicine, Kyoto University, 54 Kawahara-cho, Shogoin, Sakyo-ku, Kyoto 606-8507, Japan. E-mail: tanizawalakuhp.kyoto-u.ac.jp

ABSTRACT The clinical significance of serial changes in serum biomarkers in patients with idiopathic pulmonary fibrosis (IPF) remains to be established. This retrospective study was conducted to clarify the associations of serial changes in serum Krebs von den Lungen-6 (KL-6) and surfactant protein-D (SP-D) with changes in physiological indices and overall mortality in IPF.

The study subjects were 75 patients with IPF. The 6 month change in serum KL-6 was significantly correlated with changes in the percentage of the predicted forced vital capacity (FVC \% pred) and the percentage of the predicted diffusing capacity of the lung for carbon monoxide (\% DLCO), while the 6 month change in serum SP-D was correlated only with \% DLCO. During the mean follow-up period of 647 days, $22(29.3 \%)$ patients died. An increase in serum KL-6 over a 6 month period was a significant predictor of mortality even after adjustment for \%FVC, \% DLCO and serum KL- 6 at the baseline (hazard ratio 1.10 per $100 \mathrm{U} \cdot \mathrm{mL}^{-1}, 95 \%$ CI $1.01-1.18, \mathrm{p}=0.03$ ), whereas the 6 month increase in serum SP-D was not significant.

Serial measurements of serum KL-6 may provide additional prognostic information compared to that provided by physiological parameters in patients with IPF.

@ERSpublications

Serial changes in serum KL-6 are associated with changes in physiological variables and can predict survival in IPF http://ow.ly/hCkb30eauLg

Cite this article as: Sokai A, Tanizawa K, Handa T, et al. Importance of serial changes in biomarkers in idiopathic pulmonary fibrosis. ERJ Open Res 2017; 3: 00019-2016 [https://doi.org/10.1183/ 23120541.00019-2016].

This article has supplementary material available from openres.ersjournals.com

Received: Feb 102016 | Accepted after revision: July 012017

Support statement: The present study was supported in part by a grant from Japan's Ministry of Health, Labor, and Welfare to the Diffuse Lung Diseases Research Group, the Respiratory Failure Study Group, and the Research Program of Intractable Disease. Other support included a KAKENHI Grant-in-Aid for Young Scientists (B) awarded by the Japan Society for the Promotion of Science and grants from the Japan Intractable Diseases Research Foundation. Funding information for this article has been deposited with the Crossref Funder Registry.

Conflict of interest: Disclosures can be found alongside this article at openres.ersjournals.com

Copyright $\odot$ ERS 2017. This article is open access and distributed under the terms of the Creative Commons Attribution Non-Commercial Licence 4.0. 


\section{Introduction}

Idiopathic pulmonary fibrosis (IPF) is a fibrotic pulmonary disease that eventually leads to a fatal outcome $[1,2]$. The clinical course of IPF is essentially progressive but highly variable [3]. Although antifibrotic agents and lung transplantation have been proposed as therapeutic options in guidelines [1, 4], the benefits of antifibrotic agents for survival have not yet been established [5-7]. Thus, the precise prediction of disease progression is critical for stratifying patients at high risk for poor prognosis and determining the appropriate treatment among these evolving therapeutic options. Currently, several surrogate biomarkers for IPF have been explored with respect to predicting prognosis and evaluating treatment $[8,9]$.

Reported disease markers for IPF include physiological parameters such as forced vital capacity (FVC) and diffusing capacity of the lung for carbon monoxide (DLCO), radiological indices and blood biomarkers [3]. Among the blood biomarkers, Krebs von den Lungen-6 (KL-6) and surfactant protein-D (SP-D) have been widely used in clinical practice [10]. KL-6, also known as mucin 1, is a glycoprotein expressed on the surface of alveolar epithelial type II cells (AECIIs) and bronchiolar epithelial cells [9, 10]. SP-D is one of the surfactant proteins synthesised and secreted from AECIIs together with surfactant protein-A (SP-A) $[9,11]$. Both serum KL-6 and SP-D are associated with disease severity at the time of measurement as well as with long-term outcomes [12-15], although the significance of serial changes in these serum biomarkers remains undetermined. Conversely, in addition to their baseline values, serial changes in physiological indices can provide independent prognostic information [16]. These findings suggest that trends in serum KL-6 and SP-D may also be prognostic factors, independent of the baseline measurements.

This retrospective study was conducted to clarify the clinical significance of changes in serum KL-6 and SP-D. The associations of serial changes in these serum biomarkers with those in physiological indices and mortality were investigated to provide new insights into the roles of serum KL-6 and SP-D as surrogate biomarkers of IPF.

\section{Methods}

\section{Patient population}

This was a retrospective study that reviewed 97 consecutive patients with IPF who were followed up regularly for more than 3 months at the Dept of Respiratory Medicine at Kyoto University Hospital from February 2007 to April 2014 and whose serum KL-6 and SP-D levels were measured one or more times. IPF was diagnosed via a multidisciplinary diagnosis (MDD) approach according to the current guideline [1]. The earliest visit with serum biomarker measurements during the study period was set as the baseline (the first time point) and, if available, the following visit $6 \pm 2$ months later was set as the second time point. Patients were excluded if they had an active neoplastic disease or an acute worsening of a disease, such as an acute exacerbation of IPF, infection or congestive heart failure, at the first time point or if they had previously undergone therapeutic lung resection for pulmonary malignant disease.

Of these 97 patients, 75 were available at the second time point. Twenty were available only for the baseline but were followed up more than 6 months later, and the remaining two were censored within 6 months. As the present study aimed to examine the clinical relevance of serial changes in serum biomarkers, the 75 patients with the second time point data were exclusively used for the following analyses. In fact, the patients for whom serum biomarker measurements at the second time point were or were not available presented similar baseline data (supplementary table S1). No patients who did not have data available from the second time point died within 6 months of the baseline measurements.

The present study was approved by the Institutional Review Board, Kyoto University (approval no. R0239), and written informed consent was not required due to the retrospective nature of the study.

\section{Measurements at the first and second time points}

Clinical data were retrospectively collected from medical records. According to the inclusion criteria, all patients underwent serum KL-6 and SP-D measurements at the baseline using ECLIA (Eisai Corp., Tokyo, Japan) for KL-6 and EIA (Yamasa Shoyu Corp., Choshi, Japan) for SP-D. If available, measurements of the serum biomarkers at the second time point and standardised pulmonary function test (PFT) results (including FVC and/or DLCO) performed within 2 months of the first and second time points were also reviewed [17].

\section{Outcome evaluation}

The primary outcome was the transplant-censored overall survival time. When a patient underwent lung transplantation, the outcome was censored at the date of transplantation. 


\section{Statistical analysis}

Statistical analyses were performed using JMP 10.0 (SAS Institute Inc., Cary, NC, USA). Changes in values between the first and second time points were compared using the Wilcoxon matched-pairs signed-ranks test. Changes in \% FVC and \% DLCO were represented as absolute changes (the percentages of the predicted values at the second time point minus those at the first time point). Correlations between continuous variables were evaluated using the Spearman's rank correlation coefficient $r_{s}$. Cox proportional hazards models were used to determine the prognostic factors for survival. A receiver operating characteristic (ROC) analysis of biomarker changes was performed to determine the threshold for predicting death within the observational period. Mortality was calculated, and survival curves were generated using the Kaplan-Meier method and compared between groups using the log-rank test. All analyses were considered statistically significant when $\mathrm{p}<0.05$.

\section{Results}

\section{Patient characteristics}

Sixteen $(21.3 \%)$ of the 75 patients enrolled were diagnosed via the MDD approach as having IPF with the histopathological usual interstitial pneumonia pattern (table 1). The mean age of the study patients was 69.9 years at baseline, and $64(85.3 \%)$ patients were male. The \% FVC measurements were available for 57 patients at both time points, and their \% FVC values were $87.5 \pm 20.4 \%$ and $86.3 \pm 22.3 \%$ at the first and second time points, respectively. The absolute change in \% FVC between the two time points was $-1.2 \pm 0.8 \%$, and there was no significant difference between the two time points $(p=0.21)$. After 6 months, a significant improvement in \% FVC $(\geqslant 10 \%)$, no change in \% FVC $(>-10 \%$ and $<10 \%)$ and a significant decline in \% FVC $(<-10 \%)$ were observed in one $(2 \%), 50(88 \%)$ and six (11\%) patients, respectively. The \% DLCO measurements were available for 56 patients at both time points and a significant decrease was observed in \% DLCO, with an absolute change of $-2.4 \pm 0.8 \%(\mathrm{p}<0.01)$. After 6 months, no change in \% DLCO (changes $>-15 \%$ and $<15 \%)$ and a decline in $\%$ DLCO $(\leqslant-15 \%)$ were observed in 55 (98\%) patients and one (2\%) patient, respectively. Serum KL-6 tended to be elevated from $1121 \pm 733 \mathrm{U} \cdot \mathrm{mL}^{-1}$ to $1251 \pm 889 \mathrm{U} \cdot \mathrm{mL}^{-1}(\mathrm{p}=0.06)$ and the absolute change was $131 \pm 63 \mathrm{U} \cdot \mathrm{mL}^{-1}$. Serum SP-D did not increase significantly between the two time points $\left(302 \pm 224 \mathrm{ng} \cdot \mathrm{mL}^{-1}\right.$ at the first time point; $308 \pm 240 \mathrm{ng} \cdot \mathrm{mL}^{-1}$ at the second time point) $(\mathrm{p}=0.71)$. At baseline, $11(14.7 \%)$ patients were only receiving pirfenidone and $2(2.7 \%)$ were treated with a combination of pirfenidone and corticosteroid (CS) with or without an immunosuppressive agent (IS). Six (8.0\%) patients were only treated with CS and $5(6.7 \%)$ were treated with both CS and IS. A total of 19 (25.3\%) patients began pirfenidone between the first and second time points, but two of these 19 patients withdrew from pirfenidone therapy because of adverse effects.

\section{TABLE 1 Patient characteristics ( $n=75$ )}

\begin{tabular}{|c|c|c|c|c|}
\hline & $\begin{array}{l}\text { First time } \\
\text { point }\end{array}$ & $\begin{array}{c}\text { Second time } \\
\text { point }\end{array}$ & $\begin{array}{l}6 \text { month } \\
\text { change }\end{array}$ & p-value \\
\hline Age years & $69.9 \pm 8.7$ & & & \\
\hline Male & $64(85.3 \%)$ & & & \\
\hline $\begin{array}{l}\text { Current/previous/never } \\
\text { smoker }\end{array}$ & $\begin{array}{c}7(9.3 \%) / 62(82.7 \%) / \\
6(8.0 \%)\end{array}$ & & & \\
\hline Biopsy-proven & $16(21.3 \%)$ & & & \\
\hline Treated with pirfenidone & $11(14.7 \%)$ & $27(36.0 \%)$ & & \\
\hline Treated with CS and/or IS & $13(17.3 \%)$ & $15(20.0 \%)$ & & \\
\hline FVC $\%$ predicted & $87.5 \pm 20.4^{7}$ & $86.3 \pm 22.3^{7}$ & $-1.2 \pm 0.8$ & 0.21 \\
\hline DLco $\%$ predicted & $43.5 \pm 11.1^{+}$ & $41.1 \pm 13.2^{+}$ & $-2.4 \pm 0.8$ & $<0.01$ \\
\hline CPI & $47.4 \pm 10.7^{+}$ & $49.2 \pm 13.0^{+}$ & $1.7 \pm 0.7$ & 0.03 \\
\hline Serum KL-6 U.mL $\mathrm{mL}^{-1}$ & $1121 \pm 733$ & $1251 \pm 889$ & $131 \pm 63$ & 0.06 \\
\hline Serum SP-D ng.mL ${ }^{-1}$ & $302 \pm 224$ & $308 \pm 240$ & $7 \pm 18$ & 0.71 \\
\hline
\end{tabular}

Data are presented as mean \pm SD unless otherwise stated. CS: corticosteroid; IS: immunosuppressive agent; FVC: forced vital capacity; DLCo: diffusing capacity of the lung for carbon monoxide; CPI: composite physiologic index. \#: comparisons between the first and second time points using the Wilcoxon matched-pairs signed-ranks test; KL-6: Krebs von den Lungen-6; SP-D: surfactant protein-D. ๆ: $n=57$, a subject was included if FVC data were available at both time points; ${ }^{+}: n=56$, a subject was included if $D$ LCo and CPI data were available at both time points. 


\begin{tabular}{|c|c|c|c|c|}
\hline & \multicolumn{2}{|c|}{$\begin{array}{l}6 \text { month change in } \\
\text { FVC } \% \text { predicted }\end{array}$} & \multicolumn{2}{|c|}{$\begin{array}{l}6 \text { month change in } \\
\text { DLco \% predicted }\end{array}$} \\
\hline & $r_{s}$ & p-value & $r_{s}$ & p-value \\
\hline \multicolumn{5}{|l|}{ First time point } \\
\hline Serum KL-6 & -0.25 & 0.07 & -0.13 & 0.36 \\
\hline Serum SP-D & -0.09 & 0.51 & -0.07 & 0.63 \\
\hline \multicolumn{5}{|l|}{6 month change } \\
\hline Serum KL-6 & -0.38 & $<0.01$ & -0.33 & 0.01 \\
\hline Serum SP-D & -0.37 & $<0.01$ & -0.14 & 0.32 \\
\hline
\end{tabular}

\section{Correlations between serum biomarkers and physiological parameters}

At baseline, serum KL-6 was correlated with $\%$ DLCO $\left(r_{s}=-0.34, p<0.01\right)$ but was not correlated with $\%$ FVC $\left(\mathrm{r}_{\mathrm{s}}=-0.20, \mathrm{p}=0.10\right)$. Serum SP-D was significantly correlated with \% FVC and \% DLCO (\% FVC, $\mathrm{r}_{\mathrm{s}}=-0.32, \mathrm{p}<0.01 ; \%$ DLCO, $\left.\mathrm{r}_{\mathrm{s}}=-0.28, \mathrm{p}=0.03\right)$.

Correlations of the first time point value and the 6 month change in serum KL-6 or SP-D with the 6 month change in \% FVC or \% DLCO are shown in table 2. At the first time point, neither serum KL-6 nor SP-D was significantly correlated with the 6 month change in \% FVC or \% DLCO. Correlations of biomarker changes with the PFT results are also shown in table 2 . The change in serum KL-6 significantly correlated with those in \% FVC and \% DLCO $\left(\mathrm{r}_{\mathrm{s}}=-0.38, \mathrm{p}<0.01 ; \mathrm{r}_{\mathrm{s}}=-0.33, \mathrm{p}=0.01\right.$, respectively). The change in serum SP-D was also significantly associated with that in $\% \mathrm{FVC}\left(\mathrm{r}_{\mathrm{s}}=-0.37, \mathrm{p}<0.01\right)$, while the change in serum SP-D was not correlated with that in $\%$ DLCO $\left(\mathrm{r}_{\mathrm{s}}=-0.14, \mathrm{p}=0.32\right)$.

\section{Predictors for overall survival}

During the observation period, which was an average of 647 days (range, 127-2019 days), 22 (29.3\%) patients died. The causes of death were acute exacerbation in 8 (36.4\%) patients, chronic respiratory failure in $4(18.2 \%)$, pulmonary infection in $4(18.2 \%)$, lung cancer in $1(4.5 \%)$, and other causes not associated with pulmonary diseases in $4(18.2 \%)$. One patient (4.5\%) died of an unknown cause. Only two patients underwent lung transplantation.

TABLE 3 Univariate regression analyses of overall mortality using Cox proportional hazards models

\begin{tabular}{llc}
\hline Variables & HR $(95 \%$ CI) & p-value \\
\hline Baseline & & \\
Age & $1.03(0.97-1.10)$ & 0.37 \\
Male & $1.57(0.52-6.78)$ & 0.46 \\
Treated with pirfenidone & $1.13(0.26-3.39)$ & 0.84 \\
Treated with CS and/or IS & $2.09(0.66-5.78)$ & 0.20 \\
FVC \% predicted & $0.96(0.93-0.99)$ & $<0.01$ \\
DLCo \% predicted & $0.91(0.85-0.96)$ & $<0.01$ \\
CPI & $1.12(1.05-1.21)$ & $<0.01$ \\
Serum KL-6 $100 \mathrm{U} \cdot \mathrm{mL}^{-1}$ & $1.02(0.96-1.06)$ & 0.51 \\
Serum SP-D $10 \mathrm{ng} \cdot \mathrm{mL}^{-1}$ & $1.01(0.99-1.02)$ & 0.39 \\
6 month change & & 0.03 \\
FVC \% predicted & $0.89(0.80-0.99)$ & 0.85 \\
DLCo \% predicted & $0.99(0.90-1.12)$ & 0.02 \\
Serum KL-6 $100 \mathrm{U} \cdot \mathrm{mL}^{-1}$ & $1.08(1.02-1.14)$ & 0.07 \\
Serum SP-D $10 \mathrm{ng} \cdot \mathrm{mL}^{-1}$ & $1.03(1.00-1.05)$ & \\
\hline HR: hazard ratio; CS: corticosteroid; IS: immunosuppressive agent; FVC: forced vital capacity; DLCo: \\
diffusing capacity of the lung for carbon monoxide; CPI: composite physiological index; KL-6: Krebs von \\
den Lungen-6; SP-D: surfactant protein-D.
\end{tabular}


The results of the survival analyses using univariate Cox proportional hazards models are shown in table 3. $\%$ FVC and \% DLCO at baseline and the serial changes in serum KL-6 and \% FVC conferred significant risks for mortality (\% FVC at baseline, hazard ratio (HR) $0.96,95 \%$ CI $0.93-0.99, \mathrm{p}<0.01$; \% DLCO at baseline, HR 0.91, 95\% CI 0.85-0.96, p<0.01; 6 month change in serum KL-6, HR 1.08 per $100 \mathrm{U} \cdot \mathrm{mL}^{-1}$, 95\% CI $1.02-1.14, \mathrm{p}=0.02$; 6 month change in \% FVC, HR 0.89, 95\% CI $0.80-0.99, \mathrm{p}=0.03$ ). Age and treatment with pirfenidone at baseline were not associated with survival. Several multivariate Cox proportional hazard models were constructed (table 4). When the baseline and serial values of serum KL-6 and the PFT results were entered into the KL-6 model (table 4), the baseline \% FVC and \% DLCO and the change in serum KL-6 remained significant prognostic factors (\% FVC, HR 0.97, 95\% CI 0.94-1.00, $\mathrm{p}=0.047 ; \%$ DLCO, HR 0.91, 95\% CI 0.85-0.97, $\mathrm{p}<0.01 ; 6$ month change in serum KL-6, HR 1.10 per $100 \mathrm{U} \cdot \mathrm{mL}^{-1}, 95 \%$ CI 1.01-1.18, p=0.03) (table 4A, model 1). However, when the 6 month change in \% FVC was added to the model, the 6 month change in serum KL-6 was not a significant prognostic factor. When the composite physiologic index (CPI) [18] and serum KL-6 at baseline and the 6 month change in serum KL- 6 were entered into the model, the CPI at baseline and the 6 month change in serum KL- 6 were significant prognostic factors (model 2). When the baseline and serial values of serum SP-D and the PFT results were entered into the SP-D model (table 4B), neither serum SP-D at baseline nor the 6 month change in serum SP-D were significant prognostic factors.

According to these results, a ROC analysis was also performed to determine the optimal cut-off value of the 6 month change in serum KL-6 for predicting overall mortality during the observational period. The area under the curve was 0.60 , with a threshold value of $140 \mathrm{U} \cdot \mathrm{mL}^{-1}$. Survival curves of patients with 6 month changes in serum KL- $6 \geqslant 140 \mathrm{U} \cdot \mathrm{mL}^{-1}$ and $<140 \mathrm{U} \cdot \mathrm{mL}^{-1}$ were generated by the Kaplan-Meier method and are shown in figure 1 . The log-rank test demonstrated a significantly worse prognosis in patients with 6 month changes in serum KL-6 $\geqslant 140 \mathrm{U} \cdot \mathrm{mL}^{-1}(\mathrm{p}<0.01)$. The estimated 1 year transplant-censored survival rates of patients with serum KL-6 $\geqslant 140 \mathrm{U} \cdot \mathrm{mL}^{-1}$ and $<140 \mathrm{U} \cdot \mathrm{mL}^{-1}$ were $76.9 \%$ and $93.5 \%$, respectively, and the estimated 3-year transplant- censored survival rates of these patients were $25.4 \%$ and $86.4 \%$, respectively.

When $\%$ FVC $<70 \%$, \% DLCO $<40 \%$ and KL-6 $>1000 \mathrm{U} \cdot \mathrm{mL}^{-1}$ at baseline and a 6 month change in serum $\mathrm{KL}-6 \geqslant 140 \mathrm{U} \cdot \mathrm{mL}^{-1}$ were entered into a multivariate model for survival analysis as categorical variables, $\%$ DLCO $<40 \%$ and 6 month change in serum KL-6 $\geqslant 140 \mathrm{U} \cdot \mathrm{mL}^{-1}$ were significant predictors for mortality (\%DLCO $<40 \%$ at baseline, HR $4.94,95 \%$ CI $1.03-36.5, \mathrm{p}=0.046 ; 6$ month change in serum $\mathrm{KL}-6 \geqslant 140 \mathrm{U} \cdot \mathrm{mL}^{-1}$, HR 3.95, 95\% CI 1.20-14.9, p=0.02) (supplementary table S2).

\begin{tabular}{|c|c|c|c|c|}
\hline \multirow[t]{2}{*}{ Variables } & \multicolumn{2}{|c|}{ Model 1} & \multicolumn{2}{|c|}{ Model 2} \\
\hline & HR $(95 \% \mathrm{CI})$ & p-value & $\operatorname{HR}(95 \% \mathrm{CI})$ & p-value \\
\hline \multicolumn{5}{|l|}{ KL-6 model } \\
\hline \multicolumn{5}{|l|}{ Baseline } \\
\hline FVC \% predicted & $0.97(0.94-1.00)$ & 0.047 & & \\
\hline DLco $\%$ predicted & $0.91(0.85-0.97)$ & $<0.01$ & & \\
\hline $\mathrm{CPI}$ & & & $1.13(1.06-1.22)$ & $<0.01$ \\
\hline Serum KL-6 $100 \mathrm{U} \cdot \mathrm{mL}^{-1}$ & $0.98(0.89-1.05)$ & 0.66 & $0.99(0.90-1.05)$ & 0.78 \\
\hline 6 month change & & & & \\
\hline Serum KL-6, $100 \mathrm{U} \cdot \mathrm{mL}^{-1}$ & $1.10(1.01-1.18)$ & 0.03 & $1.10(1.02-1.18)$ & 0.02 \\
\hline \multicolumn{5}{|l|}{ SP-D model } \\
\hline \multicolumn{5}{|l|}{ Baseline } \\
\hline FVC \% predicted & $0.97(0.93-1.00)$ & 0.04 & & \\
\hline D Lco $\%$ predicted & $0.94(0.88-1.00)$ & 0.054 & & \\
\hline $\mathrm{CPI}$ & & & $1.12(1.05-1.21)$ & $<0.01$ \\
\hline Serum SP-D $10 \mathrm{ng} \cdot \mathrm{mL}^{-1}$ & $1.01(0.99-1.03)$ & 0.21 & $1.01(0.99-1.03)$ & 0.15 \\
\hline 6 month change & & & & \\
\hline Serum SP-D $10 \mathrm{ng} \cdot \mathrm{mL}^{-1}$ & $1.03(0.99-1.06)$ & 0.16 & $1.03(0.99-1.06)$ & 0.12 \\
\hline
\end{tabular}

HR: hazard ratio; KL-6: Krebs von den Lungen-6; FVC: forced vital capacity; DLCo: diffusing capacity of the lung for carbon monoxide; CPI: composite physiologic index; SP-D: surfactant protein-D. 


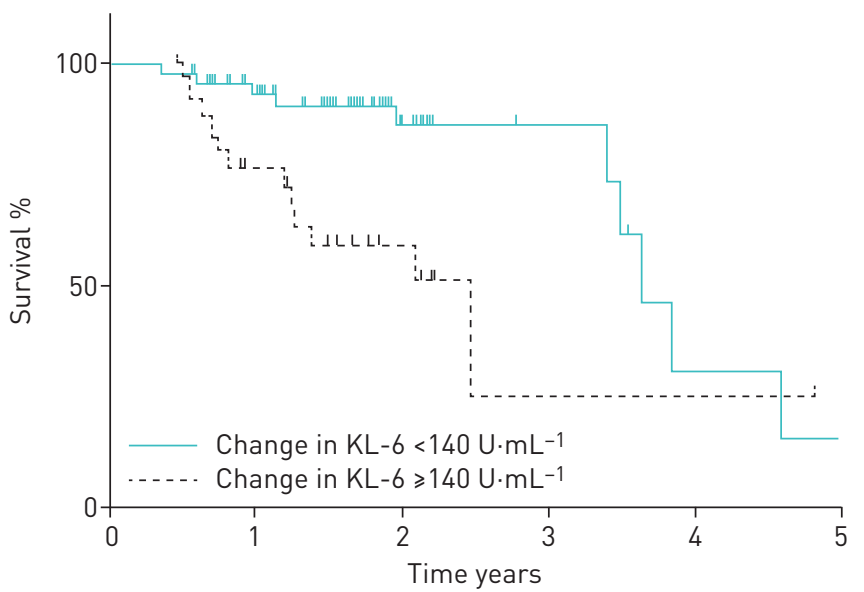

FIGURE 1 Comparison of survival between patients with $\mathrm{KL}-6 \geqslant 140 \mathrm{U} \cdot \mathrm{mL}^{-1}$ and $<140 \mathrm{U} \cdot \mathrm{mL}^{-1}$ ( $\mathrm{p}<0.01$, as shown by the log-rank test). KL-6: Krebs von den Lungen-6.

\section{Discussion}

In the present study, lower \% FVC and \% DLCO baseline values and increased 6 month serum KL-6 values in patients with IPF significantly increased the risk of mortality. The serial change in serum KL- 6 was significantly correlated with those in \% FVC and \% DLCO and was a stronger predictor of mortality than the serum KL-6 value measured at a single time point. On the other hand, neither serum SP-D at baseline nor the 6 month change in serum SP-D was associated with overall survival.

Several variables have been reported to predict survival in IPF [3]. These predictors of mortality in IPF often represent the severity of disease at the time of assessment and include the following: physiological impairment (\% FVC, \% DLCO and distance and oxygen saturation during a 6 min walk test) [3, 19-23], disease extent (high-resolution computed tomography (HRCT) indices) [24, 25] and subjective burden (dyspnoea scale) [16]. Another prognostic factor of IPF is the disease activity, or the rate of disease progression, which is assessed by the following variables: serial changes in \% FVC, \% DLCO [16, 22], HRCT indices [26] and dyspnoea score [3]. Several survival analyses have revealed that severe disease at the time of diagnosis and subsequent rapid progression significantly increase the risk of mortality in IPF [3]. Regarding physiological parameters, the impact of their serial changes (activity) may be superior to that of their baseline values (severity) $[4,16,22]$.

KL-6 is a sialylated sugar chain and is highly expressed on the cell surface of regenerating AECIIs in patients with IPF $[10,27,28]$. The elevation in serum KL-6 may result from enhanced alveolar-capillary permeability and the regeneration of AECII induced by alveolar epithelial injuries [29], both of which have been proposed as mechanisms of the pathogenesis of IPF $[2,9,30,31]$. KL-6 also promotes human pulmonary fibroblast migration and proliferation [32]. These findings collectively indicate that serum KL-6 can act as a surrogate marker for the active process of disease progression. Thus, the prognostic value of serum KL-6 for survival in IPF has been examined in several studies. SAтон et al. [12] and Yокоуама et al. [15] independently demonstrated that higher levels of serum KL-6 significantly predicted higher mortalities in patients with IPF and those with idiopathic interstitial pneumonias, respectively. However, a recent Japanese epidemiological study of IPF revealed that age, \% FVC and \% DLCO were significant predictors of mortality, while serum KL-6 was not [33]. Another study also showed that serum KL-6 weakly predicted mortality in patients with IPF but had no additional value regarding clinical parameters, including age, FVC, DLCO and change in FVC over 6 months [34], indicating a limited role of serum KL-6 as a biomarker for IPF. These controversial results may be explained by the following. First, serum KL-6 is not a specific biomarker for IPF. Cancer and acute respiratory events, such as Pneumocystis jirovecii pneumonia and acute exacerbations (AEs) of IPF, can result in increased serum KL-6 levels and thus complicate the interpretation of serum KL-6 measurements [35-38]. Second, although alveolar epithelial injury is among the major pathogenic factors of IPF, its extent, which can be represented by serum KL-6, may not be a prognostic factor for IPF [30]. Elevated serum KL-6 has been reported in other interstitial lung diseases, such as summer-type hypersensitivity pneumonitis [27, 39], and does not necessarily mean that fibrosis or aberrant repair occurred after alveolar epithelial injury. Third, as shown in the present study, serum KL-6 at a single time point could not predict the changes in \% FVC or \% DLCO over the next 6 months. A single measurement of serum KL- 6 cannot be substituted for serial measurements of \% FVC and $\%$ DLCO. 
Conversely, it is notable that the serial increase in serum KL-6 was an independent prognostic factor from the baseline physiological and serological parameters in the present study. The clinical relevance of serial changes in serum KL-6 has only been addressed in one previous study [37]. YoKoyama et al. [37] reported that increased serum KL-6 after high-dose corticosteroid therapy conferred a significant risk of mortality in patients with AEs of IPF. The present study is the first revealing the significant prognostic value of the 6 month change in serum KL-6 in the chronic phase of IPF, which could be comparable with those of physiological indices. Our results suggest that the serial monitoring of serum KL-6 in patients with IPF may provide additional prognostic information compared to baseline indices, even when repeated PFTs are unavailable.

SP-D is a lipoprotein complex synthesised and secreted from AECIIs that constitutes a liquid layer on the alveolar epithelium $[9,11]$. Elevation in serum SP-D may result from alveolar epithelial injury and the breakdown and accumulation of ACEIIs [9]. Thus, serum SP-D can represent the epithelial injury-related regeneration of ACEIIs as well as serum KL-6 and has thus been examined as a blood biomarker for IPF. The prognostic value of serum SP-D for mortality in patients with IPF also remains to be determined [13, $14,33,34]$. The present study showed that neither serum SP-D at the baseline nor the 6 month change in serum SP-D predicted overall mortality, in contrast with serum KL-6. These results may reflect the different characters of KL-6 and SP-D as serum biomarkers for IPF. Although both biomarkers are derived from regenerating ACEIIs, discrepancies between them have been reported [40]. The discrepancies between serum KL-6 and SP-D may be due to the different sources of KL-6 and SP-D. SP-D is expressed in mature ACEIIs, whereas KL-6 is expressed in the initial phase, before SP-D is expressed [28]. SP-D is also expressed in gastrointestinal epithelial cells, whereas KL-6 is not [41]. However, given the small sample size of our study, the clinical relevance of serially monitoring serum KL-6 and SP-D should be verified in future studies with larger cohorts.

The present study has several limitations. First, the present study was a retrospective study with a small number of subjects. As the subjects were restricted to patients who underwent one or more measurements of serum biomarkers, the study population may not represent the entire IPF patient population. In particular, the serial changes in serum biomarkers may be difficult to monitor in rapidly progressive cases, although no such cases were included in this study population. Second, the study subjects did not receive uniform treatments because a recommended effective treatment was not present in the guidelines until 2014 [4]. Approximately 20\% of the patients received CS and/or IS therapy, which are no longer current standard treatments. The effects of pirfenidone and nintedanib on serum KL-6 and other biomarkers and their implications should be addressed in future studies. In addition, other blood biomarkers, such as SP-A, matrix metalloproteinase-7 and CC chemokine ligand-18 [42], were not measured because of the retrospective nature of the study, which was exclusively based on clinical data available from routine practice. Additionally, although certain patients died, the observation period was short.

Despite these limitations, our results indicate the possibility that serial measurements of serum KL-6 in patients with IPF might have prognostic value independent of baseline serum KL-6 measurements. Although serum KL-6 and SP-D have been widely used in Japan as tools in clinical practice [33], there are no validated biomarkers for predicting the prognosis and monitoring the disease course of IPF [1, 43]. Further prospective studies with larger cohorts are required to clarify whether any blood biomarker can provide information in addition to that provided by routine assessments with PFTs and radiological studies.

\section{Acknowledgements}

The authors thank Dr Yoshio Taguchi and Dr Seishu Hashimoto (Dept of Respiratory Medicine, Tenri Hospital) for help drafting the manuscript.

\section{References}

1 Raghu G, Collard HR, Egan JJ, et al. An official ATS/ERS/JRS/ALAT statement: idiopathic pulmonary fibrosis: evidence-based guidelines for diagnosis and management. Am J Respir Crit Care Med 2011; 183: 788-824.

2 King TE Jr, Pardo A, Selman M. Idiopathic pulmonary fibrosis. Lancet 2011; 378: 1949-1961.

3 Ley B, Collard HR, King TE Jr. Clinical course and prediction of survival in idiopathic pulmonary fibrosis. Am J Respir Crit Care Med 2011; 183: 431-440.

4 Raghu G, Rochwerg B, Zhang Y, et al. An official ATS/ERS/JRS/ALAT clinical practice guideline: treatment of idiopathic pulmonary fibrosis. An update of the 2011 clinical practice guideline. Am J Respir Crit Care Med 2015; 192: e3-19.

5 Aravena C, Labarca G, Venegas C, et al. Correction. Pirfenidone for idiopathic pulmonary fibrosis: a systematic review and meta-analysis. PLOS ONE 2015; 10: e0140288.

6 Canestaro WJ, Forrester SH, Raghu G, et al. Drug treatment of idiopathic pulmonary fibrosis: systematic review and network meta-analysis. Chest 2016; 149: 756-766.

7 Noble PW, Albera C, Bradford WZ, et al. Pirfenidone for idiopathic pulmonary fibrosis: analysis of pooled data from three multinational phase 3 trials. Eur Respir J 2016; 47: 243-253. 

Mol Physiol 2014; 307: L681-L691.

9 Borensztajn K, Crestani B, Kolb M. Idiopathic pulmonary fibrosis: from epithelial injury to biomarkers - insights from the bench side. Respiration 2013; 86: 441-452.

10 Ishikawa N, Hattori N, Yokoyama A, et al. Utility of KL-6/MUC1 in the clinical management of interstitial lung diseases. Respir Investig 2012; 50: 3-13.

11 Goerke J. Pulmonary surfactant: functions and molecular composition. Biochim Biophys Acta 1998; 1408: 79-89.

12 Satoh H, Kurishima K, Ishikawa H, et al. Increased levels of KL-6 and subsequent mortality in patients with interstitial lung diseases. J Intern Med 2006; 260: 429-434.

13 Greene KE, King TE Jr, Kuroki Y, et al. Serum surfactant proteins-A and -D as biomarkers in idiopathic pulmonary fibrosis. Eur Respir J 2002; 19: 439-446.

14 Takahashi H, Fujishima T, Koba H, et al. Serum surfactant proteins A and D as prognostic factors in idiopathic pulmonary fibrosis and their relationship to disease extent. Am J Respir Crit Care Med 2000; 162: 1109-1114.

15 Yokoyama A, Kondo K, Nakajima M, et al. Prognostic value of circulating KL-6 in idiopathic pulmonary fibrosis Respirology 2006; 11: 164-168.

16 Collard HR, King TE Jr, Bartelson BB, et al. Changes in clinical and physiologic variables predict survival in idiopathic pulmonary fibrosis. Am J Respir Crit Care Med 2003; 168: 538-542.

17 Miller MR, Hankinson J, Brusasco V, et al. Standardisation of spirometry. Eur Respir J 2005; 26: 319-338.

18 Wells AU, Desai SR, Rubens MB, et al. Idiopathic pulmonary fibrosis: a composite physiologic index derived from disease extent observed by computed tomography. Am J Respir Crit Care Med 2003; 167: 962-969.

19 Heresi GA, Dweik RA. Strengths and limitations of the six-minute-walk test: a model biomarker study in idiopathic pulmonary fibrosis. Am J Respir Crit Care Med 2011; 183: 1122-1124.

20 Ley B, Ryerson CJ, Vittinghoff E, et al. A multidimensional index and staging system for idiopathic pulmonary fibrosis. Ann Intern Med 2012; 156: 684-691.

21 Nathan SD, Shlobin OA, Weir N, et al. Long-term course and prognosis of idiopathic pulmonary fibrosis in the new millennium. Chest 2011; 140: 221-229.

22 Flaherty KR, Andrei AC, Murray S, et al. Idiopathic pulmonary fibrosis: prognostic value of changes in physiology and six-minute-walk test. Am J Respir Crit Care Med 2006; 174: 803-809.

23 Lederer DJ, Arcasoy SM, Wilt JS, et al. Six-minute-walk distance predicts waiting list survival in idiopathic pulmonary fibrosis. Am J Respir Crit Care Med 2006; 174: 659-664.

24 Best AC, Meng J, Lynch AM, et al. Idiopathic pulmonary fibrosis: physiologic tests, quantitative CT indexes, and CT visual scores as predictors of mortality. Radiology 2008; 246: 935-940.

25 Ley B, Elicker BM, Hartman TE, et al. Idiopathic pulmonary fibrosis: CT and risk of death. Radiology 2014; 273: 570-579.

26 Maldonado F, Moua T, Rajagopalan S, et al. Automated quantification of radiological patterns predicts survival in idiopathic pulmonary fibrosis. Eur Respir J 2014; 43: 204-212.

27 Nobuoki K, Seishi K, Yukikazu A, et al. New serum indicator of interstitial pneumonitis activity: sialylated carbohydrate antigen KL-6. Chest 1989; 96: 68-73.

28 Ohtsuki Y, Nakanishi N, Fujita J, et al. Immunohistochemical distribution of SP-D, compared with that of SP-A and KL-6, in interstitial pneumonias. Med Mol Morphol 2007; 40: 163-167.

29 Inoue Y, Barker E, Daniloff E, et al. Pulmonary epithelial cell injury and alveolar-capillary permeability in berylliosis. Am I Respir Crit Care Med 1997; 156: 109-115.

30 Goh NS, Desai SR, Anagnostopoulos C, et al. Increased epithelial permeability in pulmonary fibrosis in relation to disease progression. Eur Respir J 2011; 38: 184-190.

31 McKeown S, Richter AG, O'Kane C, et al. MMP expression and abnormal lung permeability are important determinants of outcome in IPF. Eur Respir J 2009; 33: 77-84.

32 Ohshimo S, Yokoyama A, Hattori N, et al. KL-6, a human MUC1 mucin, promotes proliferation and survival of lung fibroblasts. Biochem Biophys Res Commun 2005; 338: 1845-1852.

33 Natsuizaka M, Chiba H, Kuronuma K, et al. Epidemiologic survey of Japanese patients with idiopathic pulmonary fibrosis and investigation of ethnic differences. Am J Respir Crit Care Med 2014; 190: 773-779.

34 Song JW, Do KH, Jang SJ, et al. Blood biomarkers MMP-7 and SP-A: predictors of outcome in idiopathic pulmonary fibrosis. Chest 2013; 143: 1422-1429.

35 Hiraga Y, Tanaka S, Haruma K, et al. Immunoreactive MUC1 expression at the deepest invasive portion correlates with prognosis of colorectal cancer. Oncology 1998; 55: 307-319.

36 Hirasawa Y, Kohno N, Yokoyama A, et al. Natural autoantibody to MUC1 is a prognostic indicator for non-small cell lung cancer. Am J Respir Crit Care Med 2000; 161: 589-594.

37 Yokoyama A, Kohno N, Hamada H, et al. Circulating KL-6 predicts the outcome of rapidly progressive idiopathic pulmonary fibrosis. Am J Respir Crit Care Med 1998; 158: 1680-1684.

38 Hamada H, Kohno N, Yokoyama A, et al. KL-6 as a serologic indicator of Pneumocystis carinii pneumonia in immunocompromised hosts. Intern Med 1998; 37: 307-310.

39 Kobayashi J, Kitamura S. KL-6: a serum marker for interstitial pneumonia. Chest 1995; 108: 311-315.

40 Matsuno Y, Satoh H, Ishikawa H, et al. Simultaneous measurements of KL-6 and SP-D in patients undergoing thoracic radiotherapy. Med Oncol 2006; 23: 75-82.

41 Hartl D, Griese M. Surfactant protein D in human lung diseases. Eur J Clin Invest 2006; 36: $423-435$.

42 Prasse A, Probst C, Bargagli E, et al. Serum CC-chemokine ligand 18 concentration predicts outcome in idiopathic pulmonary fibrosis. Am J Respir Crit Care Med 2009; 179: 717-723.

43 Vij R, Noth I. Peripheral blood biomarkers in idiopathic pulmonary fibrosis. Transl Res 2012; 159: 218-227. 